\title{
LE PEUPLEMENT ICHTYOLOGIQUE DU BASSIN DE L'YONNE
}

\author{
Ph. BOET (1), J. ALLARDI (1) et J. LEROY (2)
}

(1) CEMAGREF-DQEPP, 14 Avenue de Saint-Mandé, 75012 PARIS

(2) CSP Délégation Régionale $N^{\circ} 5$, Antenne de Dijon, 20 rue Charrue, 21000 DIJON

Reçu le 14 novembre 1990

Accepté le 8 février 1991

Received 14 November, 1990

Accepted 8 February, 1991

\section{RÉSUMÉ}

Cet article rend compte de la composition du peuplement ichtyologique du bassin de l'Yonne, affluent le plus important de la Seine. Les captures de 58 échantillons ponctuels sont analysées.

Sur le cours principal, les associations ichtyologiques sont conformes avec les zonations longitudinales bien connues (HUET, 1949 ; VERNEAUX, 1973), montrant une augmentation progressive de la richesse spécifique de l'amont vers l'aval. Le schéma général est confirmé à l'échelle du bassin tout entier, comme cela apparaît dans l'analyse des correspondances entre stations et compositions faunistiques. La distribution des poissons est en relation avec les paramètres morphométriques tels que l'altitude, la distance à la source et le rang fluvial. Néanmoins, des altérations apparaissent dans les parties amont des cours d'eau dues à la présence d'espèces d'eau calme en provenance des multiples étangs construits ces dernières années. Malgré une bonne qualité chimique d'eau, la chenalisation dans les zones inférieures conduit à une homogénéisation du courant et une réduction des zones d'eau courante. Ceci se traduit par une réduction de la richesse spécifique avec une disparition marquée des espèces rhéophiles.

31 espèces ont été capturées, et la confrontation avec des données anciennes montre que presque toutes les espèces migratrices (excepté l'anguille) ont disparu, principalement du fait de la construction des barrages pour la navigation. 5 nouvelles espèces sont dues aux introductions.

\section{THE ICHTYOFAUNA OF THE YONNE RIVER BASIN (FRANCE)}

\section{ABSTRACT}

The present paper deals with the fish composition of the Yonne River basin, one of the most important tributary of the Seine River. Catches from a total of 58 sampling points are analysed.

On the main river, fish assemblages are consistent with the well-known longitudinal zonations (HUET, 1949 ; VERNEAUX, 1973), showing a progressive increase of the specific richness from up to downstream. This general scheme is confirmed at the watershed level as described by the correspondence analysis of the stations by fauna matrix. The fish distribution is related to morphometric parameters like elevation, distance from the source and stream order. Nevertheless, disturbances appear in the upper streams, due to the presence of limnetic species escaped from the multiple new-built fish ponds. While, despite a good chemical water quality, the chenalization in the lower parts leads to a homogenization of the flow and a reduction of the lotic habitats. This results in a decrease of the species richness with a markedly disappearance of the rheophilic species.

31 species were caught, and by comparison with old data, almost all the migratory species (except anguilla) have disappeared, mainly due to the dams built for the navigation. On the contrary, 5 new species result from introduction. 


\section{INTRODUCTION}

Malgré l'importance socio-économique des ressources ichtyologiques dulçaquicoles, et bien que "les peuplements pisciaires constituent l'expression synthétique résultante, non parmi les plus sensibles mais des plus évidentes, de "l'état de santé" (GOODNIGHT, 1973) d'un système" (VERNEAUX, 1981), les synthèses sur le peuplement ichtyologique d'un même bassin hydrographique sont, en France, actuellement peu nombreuses (VERNEAUX, 1973 ; ARRIGNON, 1988 ; PATTEE, 1988).

Ainsi, les données les plus récentes concernant le bassin de l'Yonne sont dues à POPELIN (1952) qui réactualisait alors le travail de MOREAU $(1897,1898)$. Toutefois, même ces observations, par ailleurs entachées d'imprécisions liées à des problèmes de détermination spécifique et à l'appréciation subjective de l'abondance des différentes espèces, se limitent à l'Yonne moyenne ou au département de l'Yonne, respectivement.

Le présent document fait le point sur l'état actuel du peuplement de poissons de ce bassin et son évolution au cours des dernières décennies.

\section{PRÉSENTATION DU MILIEU}

L'Yonne est le plus important affluent en rive gauche de la Seine. Elle prend sa source au mont Preneley, dans le massif ancien du Morvan, à une altitude d'environ 700 mètres. Sur un parcours de 293 kilomètres, l'Yonne draine d'abord les formations cristallines du socle hercynien, puis traverse les terrains sédimentaires du Bassin Parisien (fig. 1). Ses principaux affluents (la Cure, le Serein, l'Armançon et la Vanne) se situent en rive droite.

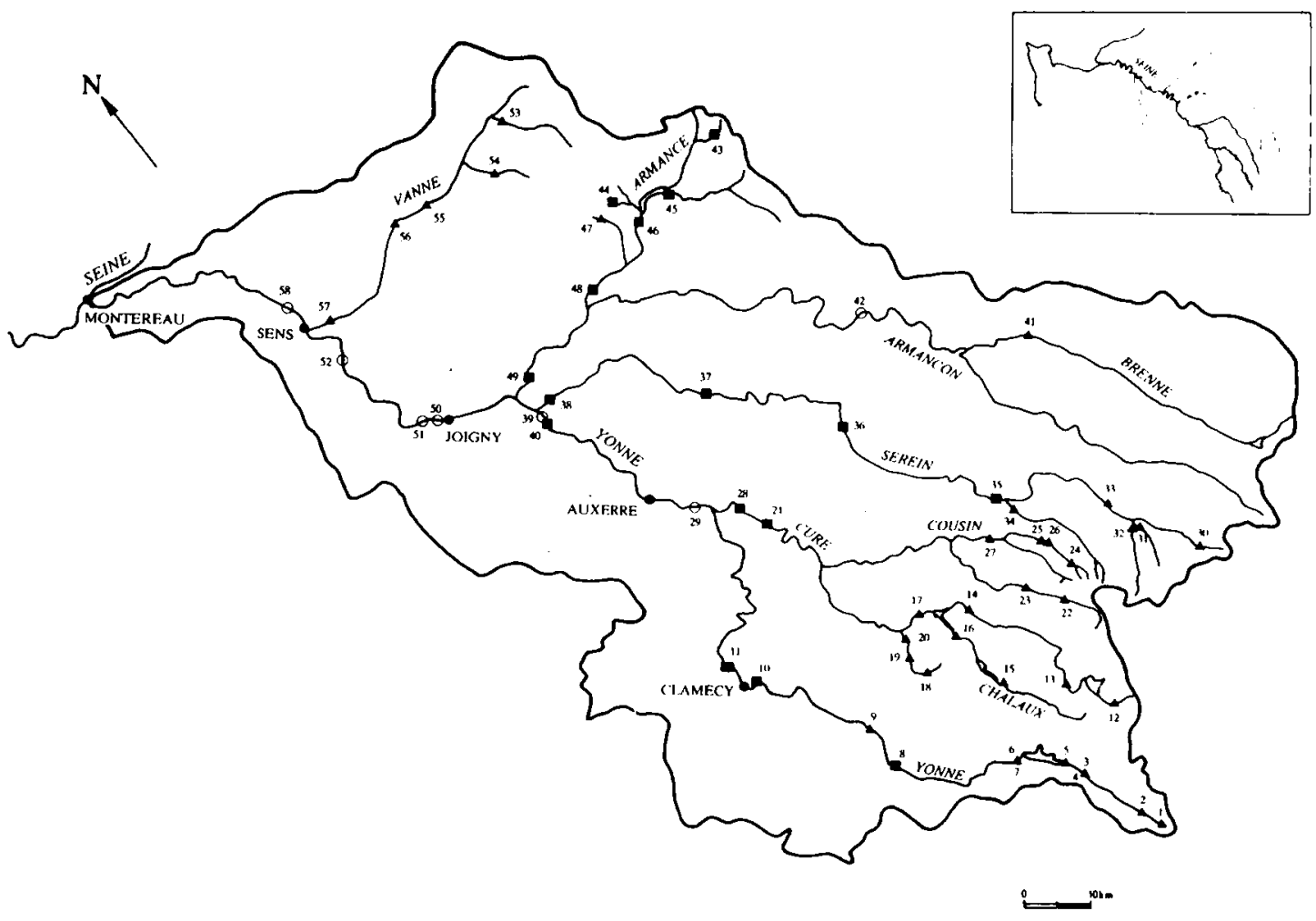

Figure 1 : Le Bassin de l'Yonne. Localisation des stations de pêche, les symboles renvoient à leur appartenance aux 3 groupes $(\Lambda: 1, \square: 2,0: 3)$ issus de la classification hiérarchique (voir dans le texte).

Figure 1 : The Yonne river basin. Location of the 58 fishing stations. Symbols refer to the 3 groups $(\Delta: 1, \square: 2,0: 3)$ steming from the clustering (see below in the text). 
Tableau 1 : Liste des stations étudiées (en gras sont indiquées les stations des pêches de juin 1988 sur le cours principal de l'Yonne).

Table 1 : List of the studied stations (in bold the 13 stations on the main river sampled during june 1988).

\begin{tabular}{|c|c|c|c|c|}
\hline ID & Stations & Cours d'eau & Code Hydro & $\mathrm{Pk}$ \\
\hline $\begin{array}{l}1 \\
2 \\
3 \\
4 \\
5 \\
6 \\
7 \\
8 \\
9 \\
10 \\
11\end{array}$ & $\begin{array}{l}\text { Source } \\
\text { Fachin les Neurots } \\
\text { Vermenou } \\
\text { Vermenou } \\
\text { Corancy } \\
\text { Montreuillon } \\
\text { Montreuillon } \\
\text { Corbigny-Pazy } \\
\text { Monceaux le Comte } \\
\text { Pont de Chevroches } \\
\text { Suray }\end{array}$ & L'Yonne & $\mathrm{H} 2 \mathrm{O}$ & $\begin{array}{l}718 \\
720 \\
724 \\
725 \\
732 \\
752 \\
754 \\
764 \\
778 \\
805 \\
817\end{array}$ \\
\hline 12 & Gien s/Cure & La Cure & $H 21020$ & 895 \\
\hline $\begin{array}{l}13 \\
14\end{array}$ & $\begin{array}{l}\text { Pont de Nataloup } \\
\text { Marigny l'Église }\end{array}$ & & & $\begin{array}{l}912 \\
932\end{array}$ \\
\hline $\begin{array}{l}15 \\
16\end{array}$ & $\begin{array}{l}\text { Brassy } \\
\text { Chalaux }\end{array}$ & Le Chalaux & $\mathrm{H} 21330$ & $\begin{array}{l}984 \\
994\end{array}$ \\
\hline 17 & St André en Morvan & La Cure & H21020 & 948 \\
\hline $\begin{array}{l}18 \\
19\end{array}$ & $\begin{array}{l}\text { Lormes } \\
\text { Empury }\end{array}$ & La Branjanne & $\mathrm{H} 21433$ & $\begin{array}{l}981 \\
993\end{array}$ \\
\hline 20 & Domecy s/Cure & & & 993 \\
\hline 21 & Asquins & La Cure & $\mathrm{H} 21020$ & 965 \\
\hline $\begin{array}{l}22 \\
23\end{array}$ & $\begin{array}{l}\text { St Aignan Cordins } \\
\text { St Léger Vauban }\end{array}$ & Le Cousin & H21523 & $\begin{array}{l}944 \\
959\end{array}$ \\
\hline 24 & La Roche en Brénil & La Romanée & $\mathrm{H} 21630$ & 983 \\
\hline $\begin{array}{l}25 \\
26\end{array}$ & $\begin{array}{l}\text { St Andeux } \\
\text { St Andeux }\end{array}$ & & & $\begin{array}{l}986 \\
991\end{array}$ \\
\hline 27 & Ste Magnance & & & 999 \\
\hline 28 & Vermenton & La Cure & H21020 & 993 \\
\hline 29 & Vaux & L'Yonne & $\mathrm{H} 2 \mathrm{O}$ & 873 \\
\hline 30 & Marcilly & Le Serein & $\mathrm{H} 23020$ & 834 \\
\hline 31 & La Motte Ternant & Le Brazon & $\mathrm{H} 23131$ & 999 \\
\hline 32 & La Motte Ternant & La Baigne & H23130 & 999 \\
\hline 33 & Vic-sous-Thil & Le Serein & H23020 & 850 \\
\hline 34 & Montberthaut & L'Argentalet & H23234 & 999 \\
\hline 35 & Vieux-Château & Le Serein & $\mathrm{H} 23020$ & 885 \\
\hline 36 & Civry s/Serein & & & 914 \\
\hline 37 & Villy-Maligny & & & 972 \\
\hline 38 & Beaumont-Bonnard & & & 996 \\
\hline 39 & Appoigny & L'Yonne & $\mathrm{H} 2 \mathrm{O}$ & 893 \\
\hline 40 & Appoigny & & & 893 \\
\hline 41 & Benoizey & La Brenne & $\mathrm{H} 24223$ & 984 \\
\hline 42 & Ancy le Franc & L'Armançon & $\mathrm{H} 24020$ & 903 \\
\hline 43 & Metz Robert & L'Armance & $\mathrm{H} 24730$ & 959 \\
\hline 44 & Auxon, Moulin blanc & Ru de la Forêt & H24734 & 992 \\
\hline 45 & Davrey & Le Landion & H24735 & 998 \\
\hline 46 & Evry la Forêt & L'Armance & $H 24730$ & 980 \\
\hline 47 & Villeneuve & Ru du Boutois & $\mathrm{H} 24740$ & 984 \\
\hline 48 & Germiny & L'Armance & $\mathrm{H} 24730$ & 996 \\
\hline 49 & Esnon & L'Armançon & $\mathrm{H} 24020$ & 992 \\
\hline 50 & Joigny & L'Yonne & $\mathrm{H} 2 \mathrm{O}$ & 914 \\
\hline 51 & Cézzy & & & 924 \\
\hline 52 & Étigny & & & 944 \\
\hline 53 & Thuisy & L'Ancre & $\mathrm{H} 26032$ & 997 \\
\hline 54 & Aix en Othe & La Nosle & $\mathrm{H} 26034$ & 995 \\
\hline 55 & St Benoit s/Vanne & La Vanne & $\mathrm{H} 26020$ & 960 \\
\hline 56 & Molinons & & & 974 \\
\hline 57 & Malay le Grand & & & 994 \\
\hline 58 & Villeperrot & L'Yonne & $\mathrm{H} 2 \mathrm{O}$ & 962 \\
\hline
\end{tabular}


Dans la partie amont de son bassin, des barrages-réservoirs (Pannecière, Crescent, Chaumeçon, Les Settons, Terre plaine Morvan...) ont été aménagés. Ils ont permis le développement de la navigation et contribuent à la régulation de la rivière au cours du cycle hydrologique annuel (lutte contre les inondations, soutien d'étiage).

Depuis très longtemps, l'Yonne est utilisée comme voie d'eau servant au transport des biens et des personnes ("train de bois", coches d'eau...). Actuellement, la navigation est possible pour des convois de 800 tonnes jusqu'à Auxerre, puis de 300 tonnes jusqu'à Clamecy. Le trafic est cependant très faible, notamment dans la partie amont, où la plaisance représente l'essentiel de la navigation. Nivernais.

L'Yonne communique avec la Saône et la Loire par les canaux de Bourgogne et du

Les plus importantes agglomérations urbaines traversées par l'Yonne, Clamecy, Auxerre, Joigny, Sens et Montereau, sont le siège d'activités économiques et industrielles qui contrastent avec le caractère très agricole du bassin versant (forêt et prairie dans le Morvan, agriculture intensive à l'aval). Cependant, la rivière et son bassin versant ne sont le siège d'aucune activité très polluante et/ou fortement consommatrice d'eau.

La qualité de l'eau est essentiellement déterminée par la nature géologique et géographique de son bassin versant ainsi que par l'activité humaine qui s'y est développée ; elle est globalement satisfaisante au regard des principaux paramètres physico-chimiques, à l'exception des différentes formes de l'azote, avec une augmentation progressive des nitrates vers l'aval. Cette bonne qualité est confirmée au plan biologique (indice diatomique et I.B.G.) (CEMAGREF, 1989).

\section{MATÉRIEL ET MÉTHODES}

\section{Les données exploitées}

Elles comprennent les résultats de pêches effectuées par le CEMAGREF sur l'axe principal de la rivière au cours du mois de juin 1988, complétés par ceux du Conseil Supérieur de la Pêche, acquis antérieurement.

Treize stations ont fait l'objet d'investigations sur l'axe principal de l'Yonne (Fig. 1 et Tab. I). Les caractéristiques géographiques et anthropiques (confluences principales, nature géologique du bassin versant, agglomérations urbaines, types d'aménagement en relation avec la navigation) susceptibles d'influencer la qualité biologique du milieu aquatique, ont guidé leur sélection.

L'échantillonnage a été réalisé classiquement par pêche à l'électricité. A pied, dans la partie amont peu profonde, où une (station 1), deux (stations 3 et 5) ou trois électrodes (stations 7, 9 et 10) ont été utilisées, en fonction de la largeur de la rivière; depuis un bateau, dans les secteurs aval, à partir de Surgy (stations 11, 29, 39, 50, 51, 52, 58).

Des estimations quantitatives, qui auraient imposé plusieurs pêches successives, n'ont pu être effectuées ; les abondances relatives des espèces sont donc à considérer avec prudence. Néanmoins, afin d'autoriser une comparaison entre les stations, une attention particulière a été portée afin que les conditions soient similaires, notamment en termes d'effort de pêche (durée comprise entre 30 et 45 minutes, une seule équipe...) et que chaque type d'habitat représenté soit prospecté pour favoriser la récolte des différentes espèces présentes. Des incertitudes subsistent toutefois, qui concernent en particulier les espèces les plus rares, puisque la sélectivité inhérente à la technique utilisée est variable selon les espèces en fonction de leur taille et de leur comportement.

Les résultats de 45 pêches, réalisées principalement par la Délégation Régionale 
$N^{\circ} 5$ du Conseil Supérieur de la Pêche (Antenne de Dijon) ont permis de compléter les données à l'échelle du bassin hydrographique de l'Yonne (Fig. 1 et Tab. I). Les protocoles mis en œuvre étant différents, en rapport avec les objectifs visés seule la présence ou l'absence de chaque espèce est considérée.

\section{Analyse des données}

- La diversité des peuplements échantillonnés sur le cours principal de l'Yonne est étudiée à l'aide des nombres de diversité proposés par HILL (1973)

$$
N_{a}=\left[\sum_{1}^{S}\left(p_{i}^{a}\right)\right]^{1 /(1 \cdot a)}
$$

où $S$ représente le nombre total d'espèces dans l'échantillon, $p_{i}$ la proportion des individus de la ie espèce, et ${ }^{a}$ l'ordre du nombre $\mathrm{N}$.

Cet indice est relativement peu sensible aux biais de l'échantillonnage car il tend à réduire l'importance des espèces rares lorsque son ordre augmente (PEET, 1974 ; ALATALO et ALATALO, 1977); ainsi, $N_{1}$ et $N_{2}$ traduisent respectivement le nombre d'espèces abondantes et le nombre d'espèces très abondantes. En d'autres termes, ces nombres effectifs d'espèces sont une mesure du nombre d'espèces dans un échantillon où chaque espèce est pondérée par son abondance (LUDWIG et REYNOLDS, 1988). Une telle "standardisation" permet alors des comparaisons robustes (DAGET, 1980).

$N$ représente aussi le nombre d'espèces, également réparties, qui permettraient d'obtenir une valeur de H' (diversité de SHANNON) égale à celle calculée pour l'échantillon. En effet, $\mathrm{N}_{\mathrm{a}}$ présente également l'avantage d'être relié à la formule d'entropie généralisée d'ordre a de RENYI (1961; cité par LEGENDRE et LEGENDRE, 1984) et il est démontré que :

$$
\begin{array}{ll}
N_{0}=S & \text { richesse spécifique } \\
N_{1}=e^{H^{\prime}} & H^{\prime}=-\sum p_{i} \ln p_{i} \text { diversité de SHANNON (1948) } \\
N_{2}=1 / \lambda & \lambda=\sum p_{1}^{2} \text { indice de SIMPSON (1949) }
\end{array}
$$

La régularité (ou équitabilité), c'est-à-dire le degré d'égalité entre les abondances des différentes espèces au sein de l'échantillon, est calculée avec l'indice proposé par MOLINARI (1989) :

$$
\begin{array}{ll}
\mathrm{G}_{2,1}=\left(\arcsin \mathrm{F}_{2.1} / 90\right) \mathrm{F}_{2,1} & \text { si } F_{2.1}>\sqrt{0.5} \\
\mathrm{G}_{2.1}=\left(\mathrm{F}_{2.1}{ }^{2}\right) \mathrm{F}_{2.1}{ }^{3} & \text { si } \mathrm{F}_{2.1}<\sqrt{0,5}
\end{array}
$$

où $F_{2,1}$ est l'indice de HILL modifié (ALATALO, 1981) :

$$
F_{2.1}=N_{2}-1 / N_{1}-1
$$

La calibration apportée par MOLINARI contribue à linéariser $F_{2.1}$ et permet une meilleure estimation de la régularité, tout en conservant une grande robustesse vis-à-vis des biais d'échantillonnage (indépendant de $S$ ). $G_{2,1}$ varie de 0 (forte dominance d'une espèce) à 1 (équi-répartition des espèces).

- Les résultats des pêches sur l'ensemble du bassin sont traités par des méthodes d'analyses multivariées, à l'aide de la programmathèque LADDAD.

Le tableau disjonctif complet, croisant les stations et les données de présenceabsence des espèces, a fait l'objet d'une analyse factorielle des correspondances multiples 
(BENZECRI et al., 1984). 26 variables actives ont été prises en compte qui correspondent aux 26 espèces ayant une fréquence d'occurrence supérieure à 2 . Les cinq espèces les plus rares ont été traitées comme variables illustratives afin d'éviter qu'elles ne prennent trop de poids dans l'analyse et ne masquent ainsi certains phénomènes sous-jacents. En outre, ont été ajoutées quatre variables illustratives caractérisant les stations et recodées en classes (ou modalités) dont les limites ont été choisies en privilégiant l'équilibre des effectifs entre les classes (Tab. II).

Tableau II : Limites de classes des variables illustratives décrivant les stations de
pêche. Table II : Limits of the classes for the illustrative variables describing the stations and used in the correspondence analysis.

\begin{tabular}{ccccc}
\hline Classes & $\begin{array}{c}\text { Distance à la source } \\
(\mathrm{km}) \text { Dis }\end{array}$ & $\begin{array}{c}\text { Altitude } \\
(\mathrm{m}) \text { Alt }\end{array}$ & $\begin{array}{c}\text { Ordre } \\
\text { Or }\end{array}$ & $\begin{array}{c}\text { Richesse sp } \\
\text { Nsp }\end{array}$ \\
\hline 1 & $<10$ & $<120$ & $1-2$ & $<5$ \\
2 & $10-50$ & $120-190$ & $3-4$ & $5-8$ \\
3 & $50-100$ & $190-340$ & $5-6$ & $8-11$ \\
4 & $>100$ & $>340$ & & $>11$ \\
\hline
\end{tabular}

L'ordre d'un tronçon de cours d'eau est le résultat d'une hiérarchisation de l'ensemble des tronçons d'un même bassin versant suivant une méthode introduite par HORTON (1945), précisée par STRAHLER (1957), puis reprise en écologie aquatique par KUEHNE (1962). Les petits cours d'eau sans tributaires sont de 1er ordre. L'ordre augmente d' 1 chaque fois que deux rivières de même ordre confluent (deux rivières d'ordre 2 donnent une rivière d'ordre 3). L'utilité de cette notion repose sur l'hypothèse préalable qu'en moyenne, pour un échantillon suffisamment grand de tronçons, les numéros d'ordre sont directement proportionnels aux dimensions relatives des bassins versants, à la dimension du lit du cours d'eau et aux débits aux stations considérées dans le réseau hydrographique (STRAHLER, 1957). En moyenne, quand l'ordre augmente, la pente et l'altitude diminuent, alors que le débit augmente. En ce sens, l'ordre rejoint certains des critères retenus par VERNEAUX $(1973,1977)$ pour le calcul du niveau typologique théorique des cours d'eau. L'ordre des cours d'eau à chaque station de pêche a été établi à partir de cartes Oro-Hydrographiques de l'IGN en considérant l'ensemble des cours d'eau permanents cartographiés à l'échelle (1:100000).

Enfin, une classification ascendante hiérarchique des stations, sur les cinq premiers facteurs de l'analyse des correspondances, a été réalisée par la méthode des voisins réciproques en utilisant le critère d'aggrégation dit du moment centré d'ordre 2 (JUAN, 1984). 
Tableau III : Liste des espèces de poissons recensées dans le bassin de l'Yonne ( ${ }^{*}$ indique les espèces introduites depuis 1899 ; "signale les espèces non présentes dans les pêches de juin 1988 sur le cours principal).

Table III : List of the species collected in the Yonne river basin ( ${ }^{*}$ indicates species introduced since 1899 ; “ denotes species which were not captured during the june 1988 sampling on the main river).

$\begin{array}{llcl}\text { Familles } & \text { Espèces } & \text { Noms communs }\end{array}$

\section{Salmonidae}

Salmo trutta fario

Oncorhynchus mikiss

(= Salmo gairdneri)

Thymallus thymallus

\section{Esocidae}

Esox lucius

Truite fario

TRF

Truite arc-en-ciel

Ombre

$T A C * \cdots$

$\mathrm{OBR}^{*}$

Brochet

BRO

\section{Cyprinidae}

Cyprinus carpio

Barbus barbus

Gobio gobio

Tinca tinca

Chondrostoma nasus

Abramis brama

Blicca bjoerkna

Rutilus rutilus

Scardinius erythrophtalmus

Alburnoides bipunctatus

Alburnus alburnus

Leuciscus cephalus

Leuciscus leuciscus

Phoxinus phoxinus

\section{Cobitidae}

Cobitis taenia

Nemacheilus barbatulus

\section{Ictaluridae}

Ictalurus melas

\section{Anguillidae}

Anguilla anguilla

\section{Gasterosteidae}

Gasterosteus aculeatus

Pungitius pungitius

\section{Gadidae}

Lota lota

\section{Centrarchidae}

Lepomis gibbosus

\section{Percidae}

Gymnocephalus cernua

Perca fluviatilis

Stizostedion lucioperca

\section{Cottidae}

Cottus gobio

Carpe commune

Barbeau fluviatile

Goujon

Tanche

Hotu

Brème

Brème bordelière

Gardon

Rotengle

Spirlin

Ablette

Chevaine

Vandoise

Vairon

CAR

BAF

GOU

TAN

HOT

BRE

BRB

GAR

ROT

SPI

$\mathrm{ABL}$

CHE

VAN

VAI

Loche de rivière

LOR

Loche franche

LOF

Poisson-chat

$\mathrm{PCH}$ * “

Anguille

ANG

Épinoche

EPI

Epinochette

EPT

Lotte

LOT

Perche soleil

PES *

Grémille

GRE

Perche

PER

Sandre

SAN *

Chabot

CHA

Petromyzonidae

Lampetra planeri

Lamproie de Planer 


\section{RÉSULTATS}

\section{Le cours principal de l'Yonne}

Au cours de la campagne de pêches électriques qui s'est déroulée du 13 au 23 juin 1988,1400 poissons ont été capturés soit une masse totale de $80,3 \mathrm{~kg}$.

Avec respectivement 39,17 et $6,5 \mathrm{~kg}$, le Chevaine, le Gardon et la Truite se situent en termes de biomasse aux trois premiers rangs. Seules cinq espèces, le Chabot, la Loche franche, le Gardon, le Chevaine et la Truite présentent chacune des effectifs supérieurs à $5 \%$ de l'ensemble des captures.

27 espèces ont été recensées (Tab. III et IV) ce qui représente une richesse spécifique élevée, conforme à la situation biogéographique de ce cours d'eau ; le maximum est observé aux stations du Pont de Chevroches et de Surgy qui comptent 14 espèces, tandis que le minimum est noté au point le plus amont, près de la source, où une seule espèce a été échantillonnée.

Le nombre d'espèces augmente depuis l'amont jusqu'à Surgy et diminue ensuite dans la partie aval (fig. 2).

Aux stations amont, la diversité s'accroît progressivement mais beaucoup d'espèces demeurent rares ; l'écart entre $N_{0}$ et $N_{1}$ est relativement élevé et la régularité, très fluctuante, reste faible (fig. 2 et $3 ;$ Tab. V).

Les stations du Pont de Chevroches et de Surgy sont les plus riches en espèces ; la qualité du peuplement est meilleure à Surgy, les abondances relatives des espèces étant mieux réparties. Au Pont de Chevroches, l'Yonne est large et encore peu profonde, la granulométrie grossière ; c'est une zone relativement calme et homogène à faible variété d'abris pour les poissons, sans faciès lotique. Au contraire, à Surgy deux faciès se distinguent : une zone lentique assez profonde, colonisée par des Nénuphars et des Myriophylles, et une zone lotique de radier à granulométrie grossière; les habitats y sont bien diversifiés.

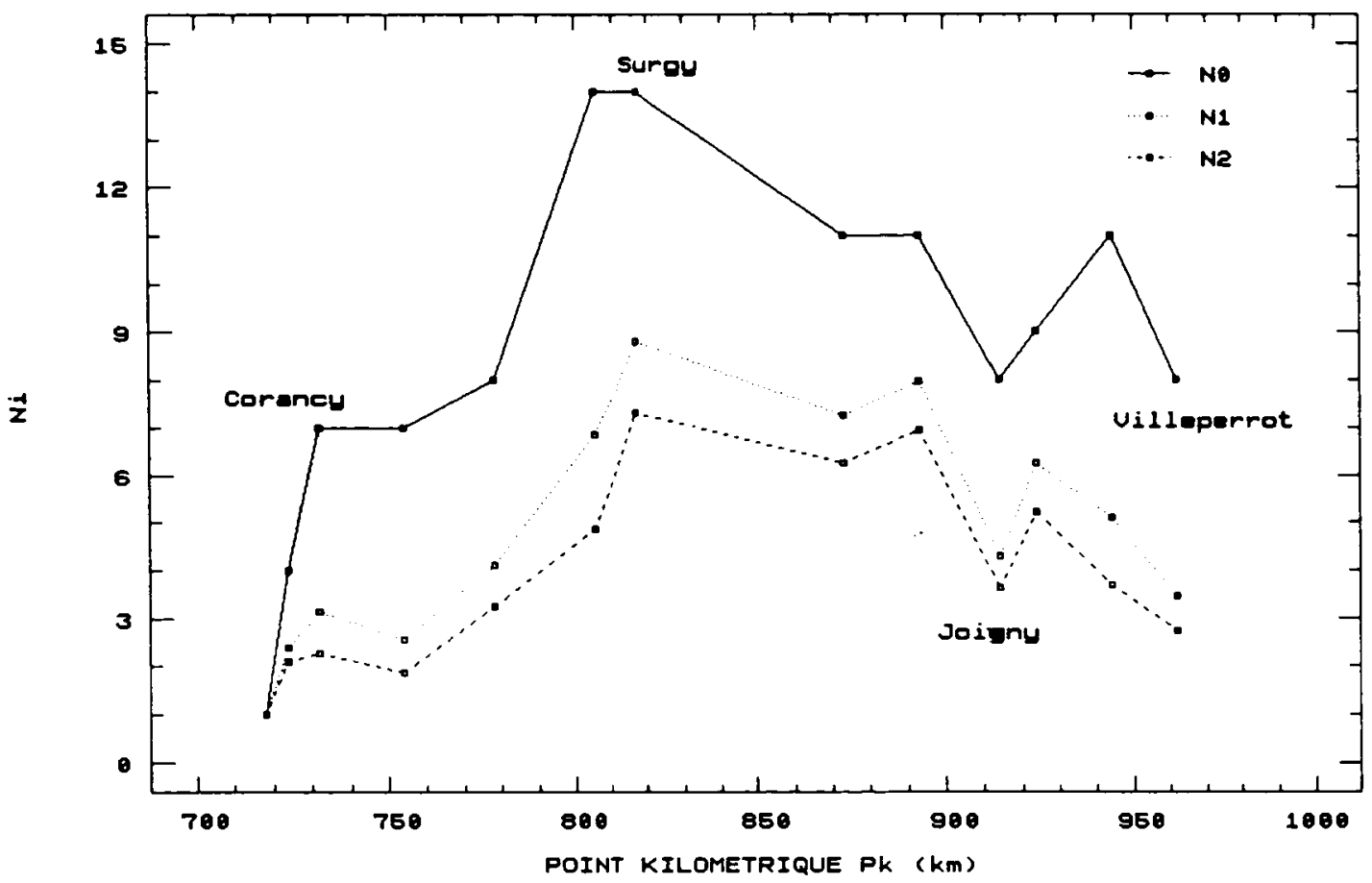

Figure 2 : Evolution des nombres de diversité le long du cours principal de l'Yonne.

Figure 2 : Hill's numbers evolution along the main Yonne river. 


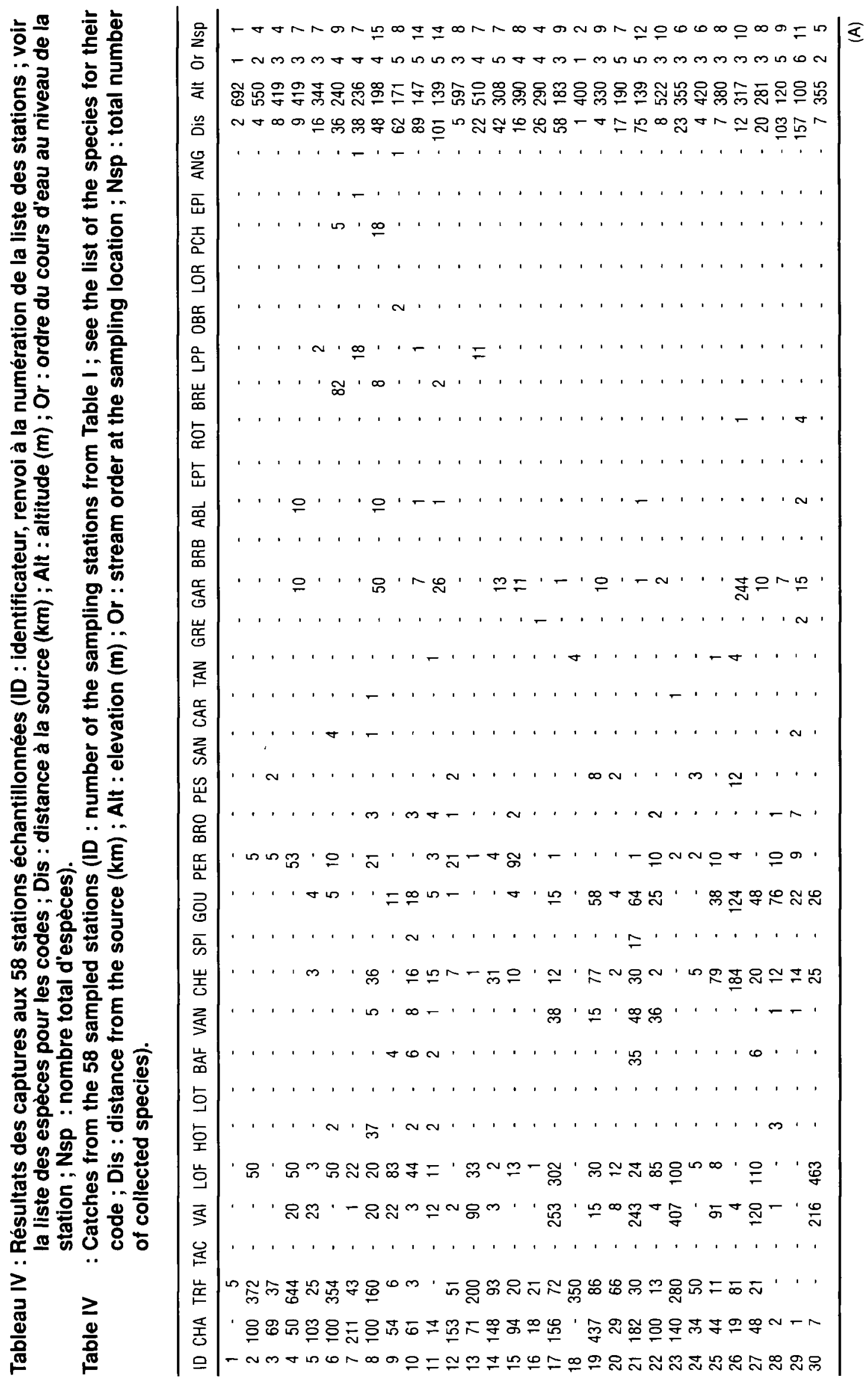




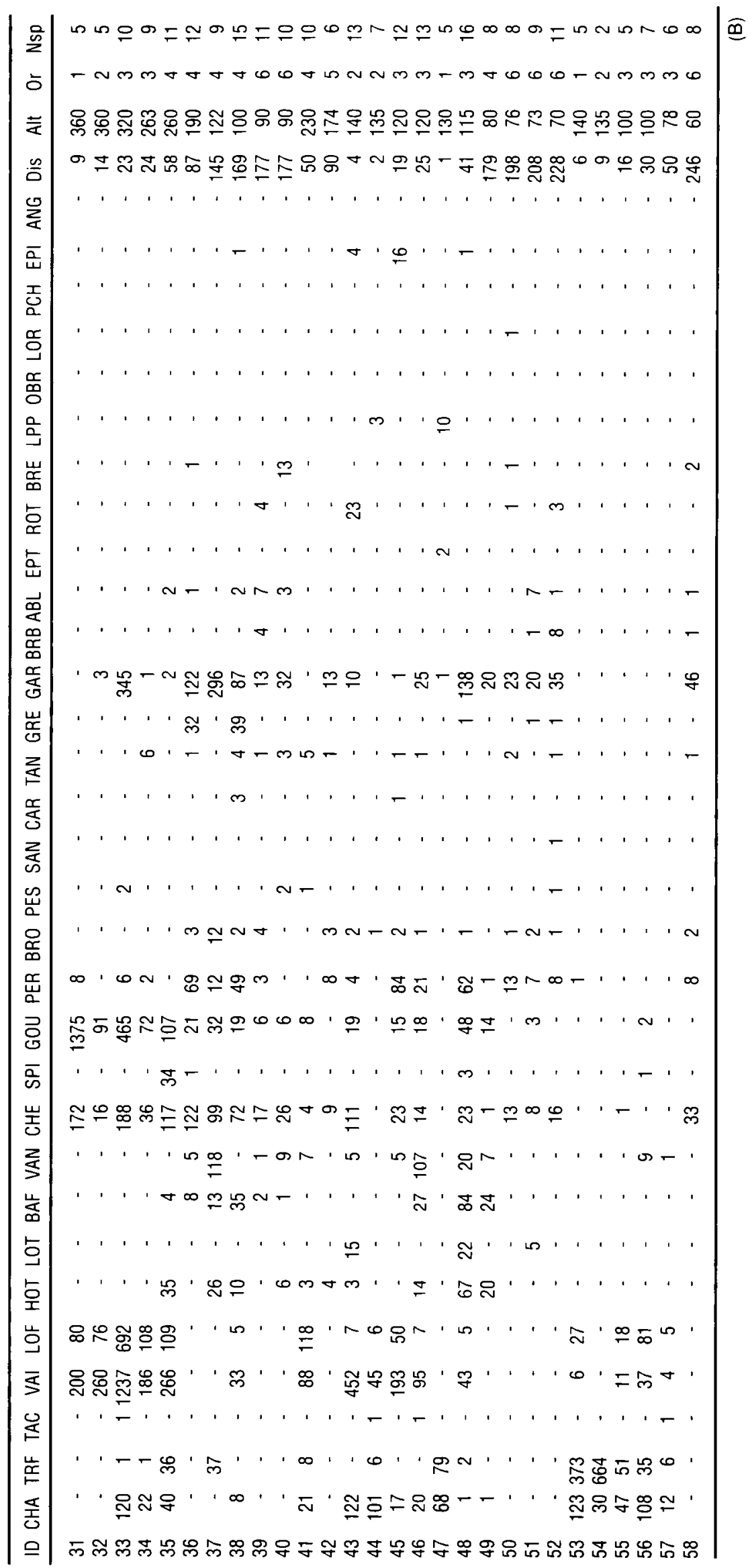


Dans la zone aval jusqu'à Appoigny, bien que le nombre total d'espèces diminue, le nombre d'espèces abondantes ne varie guère et la régularité progresse.

Ensuite, le peuplement s'appauvrit principalement aux dépens des espèces rhéophiles ; il présente des déséquilibres. Les espèces abondantes sont peu nombreuses pour ce type de cours d'eau, notamment à Joigny ou Villeperrot. La régularité est parfois très faible comme à Étigny.

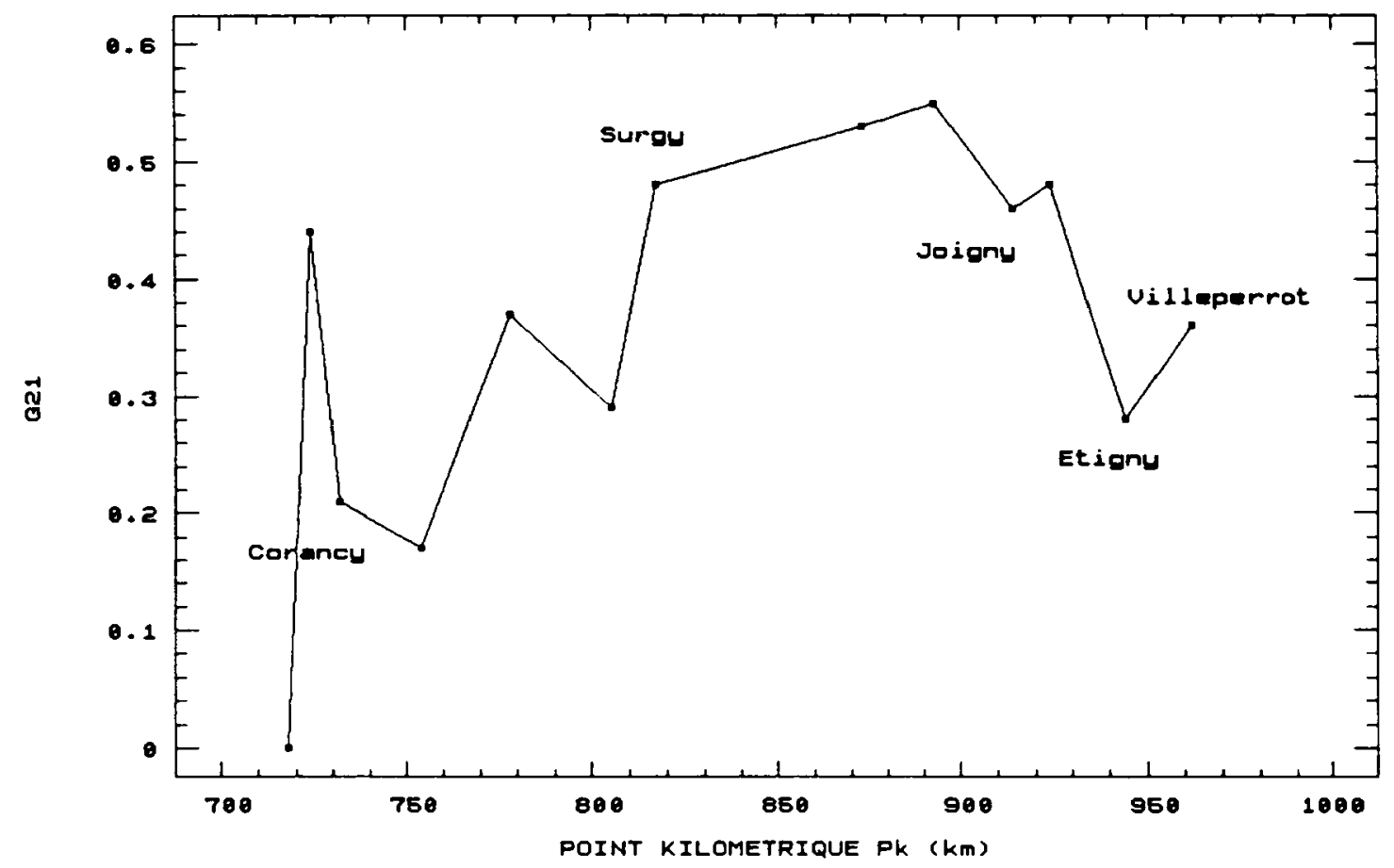

Figure 3 : Evolution de la régularité le long du cours principal de l'Yonne.

Figure 3 : Regularity evolution along the main Yonne river.

Joigny est un secteur navigué, d'une profondeur homogène d'environ 1,5 à $2 \mathrm{~m}$ en bordure de rive. Très large, il se caractérise principalement par une grande monotonie des fonds et des berges environnantes. Les habitats peu nombreux ne sont guère variés.

Cette monotonie du milieu se retrouve à des degrés divers dans les stations de la partie aval de l'Yonne et peut expliquer l'appauvrissement du peuplement piscicole.

L'évolution globale de la composition spécifique du peuplement piscicole est conforme aux schémas classiques de zonations (HUET, 1949 ; ILLIES et BOTOSANEANU, 1963) ou de typologie (VERNEAUX, 1973), lesquels fournissent une "probabilité" de présence de certains peuplements de poissons en fonction de paramètres physiques tels que la pente, la largeur et le débit. La succession zone à salmonidés à l'amont, zone intermédiaire à Barbeaux et zone à Brèmes à l'aval est en effet bien marquée.

Toutefois, la présence en zone amont (Vermenou) de la Perche et de la Perche Soleil, qui sont deux espèces normalement inféodées à des milieux plus calmes, est remarquable. Ce phénomène particulier s'explique par la dévalaison d'individus provenant d'étangs situés en amont de cette station. 
Tableau V : Évolution des nombres de diversité et de la régularité le long du cours principal de l'Yonne.

Table V : Hill's numbers and regularity evolution along the main Yonne river.

\begin{tabular}{rllllllllllllll}
\hline Stations & 1 & 3 & 5 & 7 & 9 & 10 & 11 & 29 & 39 & 50 & 51 & 52 & 58 \\
\hline $\begin{array}{r}\text { Richesse } \\
\mathrm{N}_{0}\end{array}$ & 1 & 4 & 7 & 7 & 8 & 14 & 14 & 11 & 11 & 8 & 9 & 11 & 8 \\
$\begin{array}{r}\text { Diversité } \\
\mathrm{N}_{1}\end{array}$ & 1.00 & 2.40 & 3.15 & 2.57 & 4.12 & 6.84 & 8.81 & 7.25 & 7.97 & 4.30 & 6.24 & 5.10 & 3.44 \\
$\mathrm{~N}_{2}$ & 1.00 & 2.09 & 2.27 & 1.88 & 3.24 & 4.87 & 7.31 & 6.25 & 6.95 & 3.62 & 5.22 & 3.68 & 2.74 \\
$\begin{array}{r}\text { Régularité } \\
\mathrm{G}_{2.1}\end{array}$ & 0.00 & 0.44 & 0.21 & 0.17 & 0.37 & 0.29 & 0.48 & 0.53 & 0.55 & 0.46 & 0.48 & 0.28 & 0.36 \\
\hline
\end{tabular}

\section{Le réseau hydrographique}

Les 45 résultats d'inventaires complémentaires, répartis en différents points du bassin de l'Yonne, portent à 58 le nombre total de stations étudiées et à 31 le nombre d'espèces recensées (Tab. III et IV).

Le maximum d'espèces observées simultanément (15) est atteint à Corbigny-Pazy sur l'Yonne et à Beaumont-Bonnard sur le Serein.

Les deux premiers axes factoriels de l'analyse des correspondances multiples extraient $34 \%$ de l'inertie du nuage (respectivement 21 et $13 \%$ ).

Par "construction", les modalités des descripteurs des stations (Dis, Alt et Or) évoluent conjointement dans ce plan (fig. 4) ; elles forment ainsi un gradient le long duquel se distribuent les projections des modalités-espèces. 


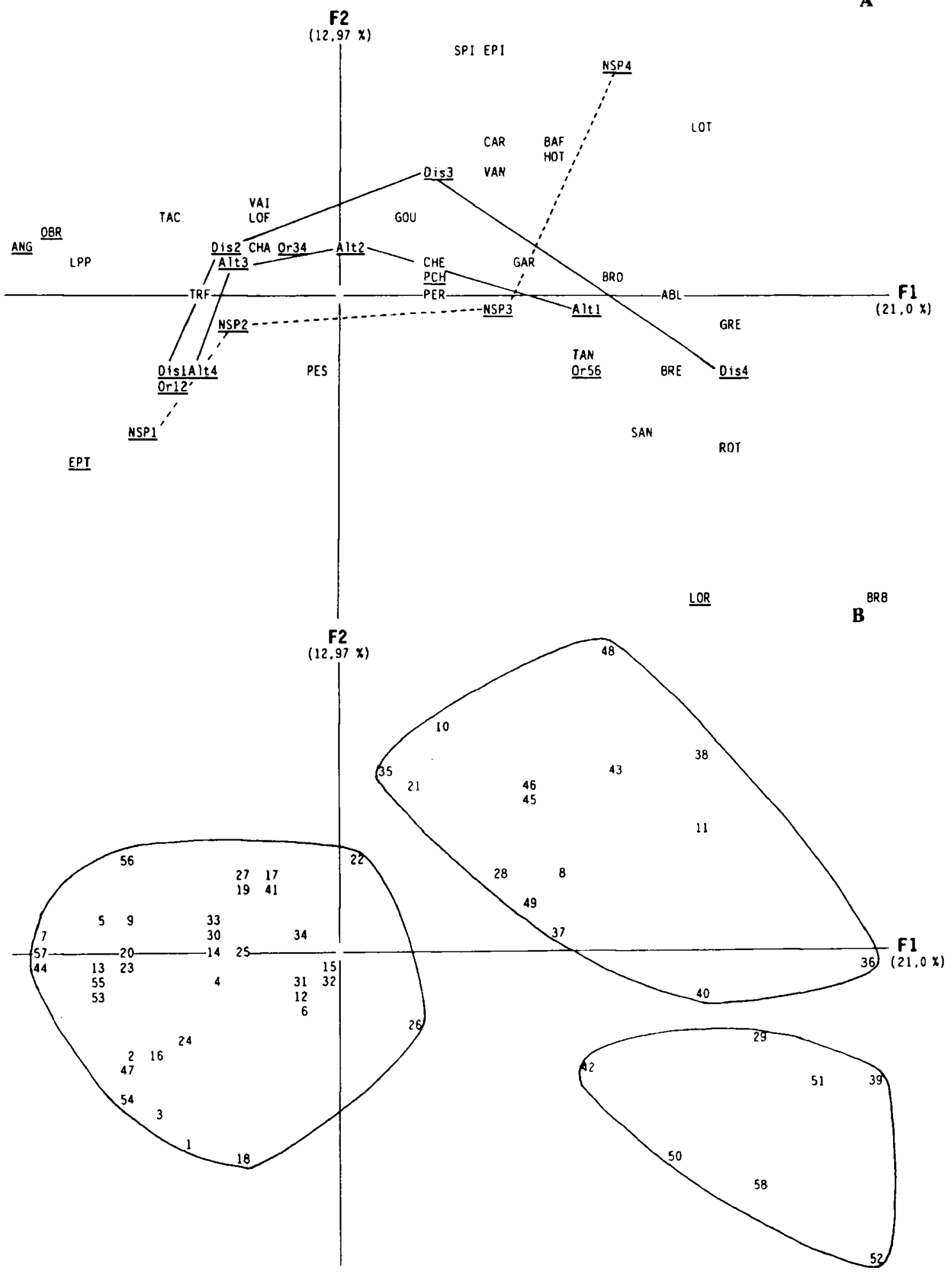

Figure 4 : Analyse factorielle des correspondances multiples ; projection des modalités présence des espèces (A) et des 58 stations (B) dans le plan des deux premiers facteurs. Représentation de la classification associée sur les stations (B). Sont soulignées les variables illustratives.

Figure 4 : Correspondence analysis. Plots of the species presence modalities (A) and of the 58 stations (B) in the two first factors plane. Representation of the associated clustering of the stations (B). Illustrative variables are underlined. 
Globalement le nombre total d'espèces capturées (Nsp) augmente régulièrement pour les stations d'ordre 1 à 4 , puis reste sensiblement constant pour les stations d'ordre 5 et 6 (voir aussi le diagramme fig. 5).

La zonation "classique" se retrouve également bien décrite sur le plan factoriel avec, dans les secteurs amont, un peuplement composé des Truite, Chabot, Vairon, Loche franche, Ombre et Lamproie de Planer ; en position médiane, la présence du Barbeau, du Hotu, de la Vandoise, du Chevaine, du Spirlin et du Goujon et, à l'aval, des Brochet, Tanche, Grémille, Sandre, Rotengle, Ablette et Brème.

Comme il a déjà été noté, certaines espèces sont observées dans des secteurs qui ne correspondent pas à leur préférendum habituel. C'est le cas de la Perche Soleil en zone amont, évoqué précédemment, ou de la Carpe qui se place ici à proximité du Barbeau, du Chevaine et de la Vandoise. Ces phénomènes rendent compte des perturbations locales de la distribution des espèces.

La classification hiérarchique des stations, sur les cinq premiers facteurs extraits par l'analyse factorielle des correspondances multiples, permet une partition en trois groupes (fig. 4).

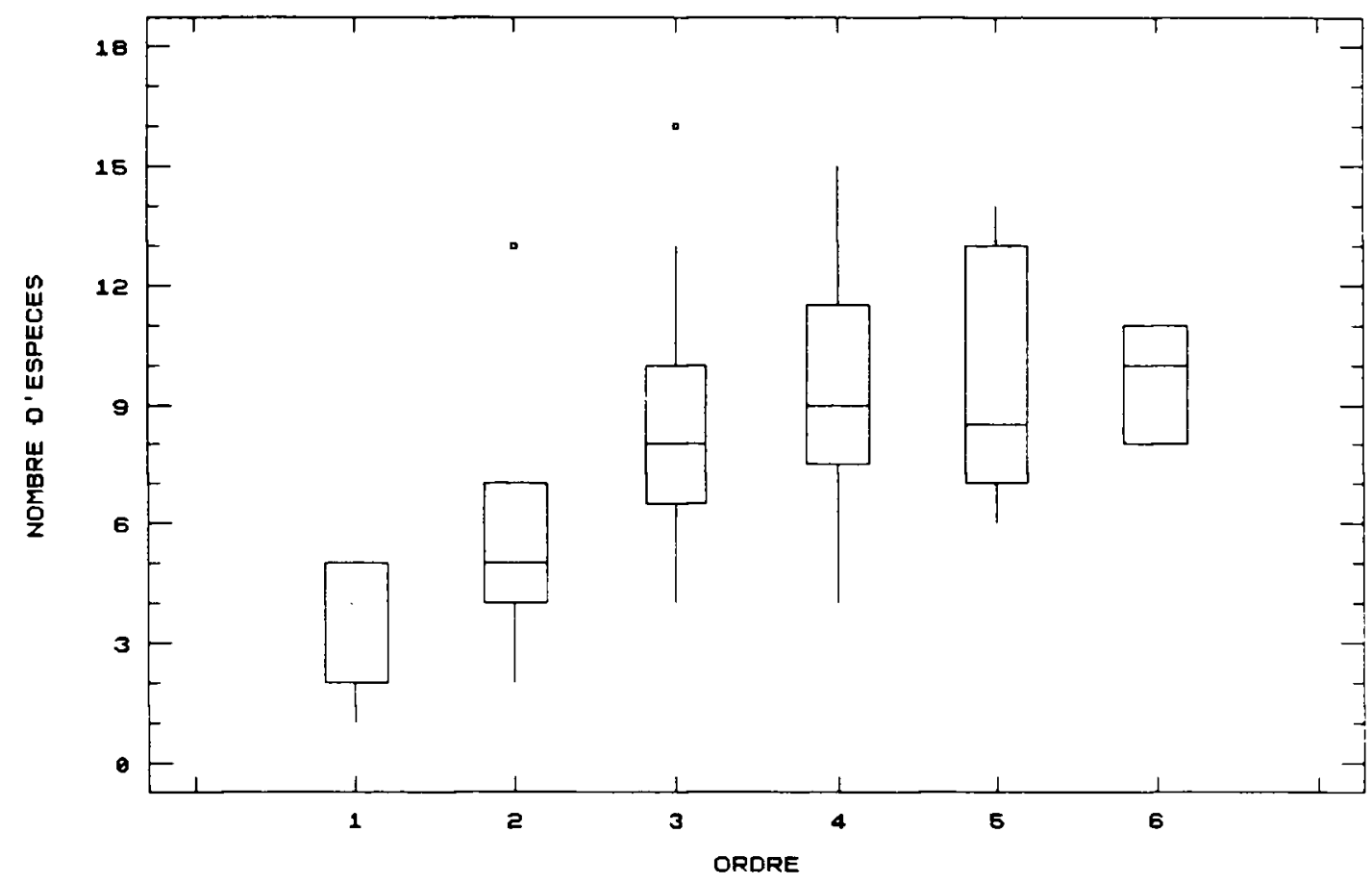

Figure 5 : Evolution du nombre d'espèces capturées en fonction de l'ordre du cours d'eau au niveau des 58 stations étudiées.

Légende des diagrammes boîte-à-moustaches : la boîte centrale couvre les valeurs comprises entre les $1^{\text {er }}$ et $3^{\mathrm{e}}$ quartiles (50\% de l'ensemble des données), les moustaches s'étendent entre les extrêmes (minimum et maximum), tandis que la ligne centrale représente la médiane. Les valeurs s'écartant de plus de 1,5 fois la longueur de la boîte (intervalle interquartile) sont tracées individueltement.

Figure 5 : Number of species vs stream order for the 58 fishing stations.

Legend of Box-and-Wisker plots : the central box covers the middle $50 \%$ of the data values, between the lower and upper quartiles. The whiskers extend out to the extremes (minimum and maximum values), while the central line is at the median. Values that are without 1.5 times the interquartiles range are ploted as separate points. 
Le premier groupe rassemble les stations situées en tête de bassin dans des secteurs peu perturbés par des aménagements, le deuxième se compose de sites correspondant principalement à des secteurs court-circuités ou à des secteurs de rivières de moyenne importance, et le troisième à des secteurs de larges rivières de plaine chenalisées et naviguées (fig. 1). Les diagrammes de la figure 6 illustrent l'évolution des variables mésologiques caractérisant ces trois ensembles, ainsi que la distribution des espèces en leur sein.
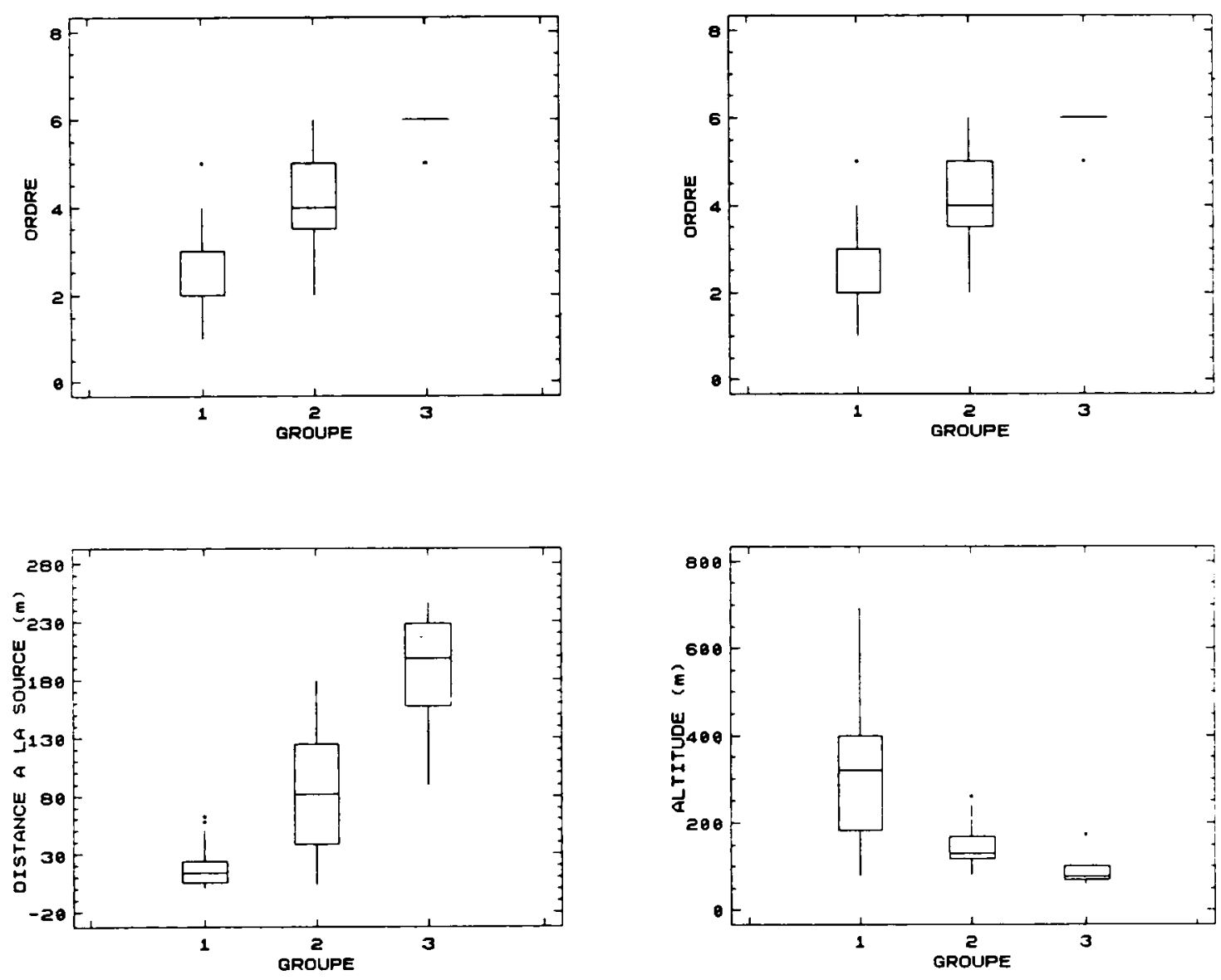

Figure 6 : Caractérisation des 3 groupes de stations issus de la classification hiérarchique.

Legende des diagrammes boite-à-moustaches : la boite centrale couvre les valeurs comprises entre les $1^{\text {er }}$ et $3^{\text {e }}$ quartiles (50\% de l'ensemble des données), les moustaches s'étendent entre les extrêmes (minimum et maximum), tandis que la ligne centrale représente la médiane. Les valeurs s'écartant de plus de 1,5 fois la longueur de la boîte (intervalle interquartile) sont tracées individuellement.

Figure 6 : Characterization of the groups of stations steming from the clustering. Legend of Box-and-Wisker plots : the central box covers the middle $50 \%$ of the data values, between the lower and upper quartiles. The whiskers extend out to extremes (minimum and maximum values), while the central line is at the median. Values that are without 1.5 times the interquartiles range are ploted as separate points. 

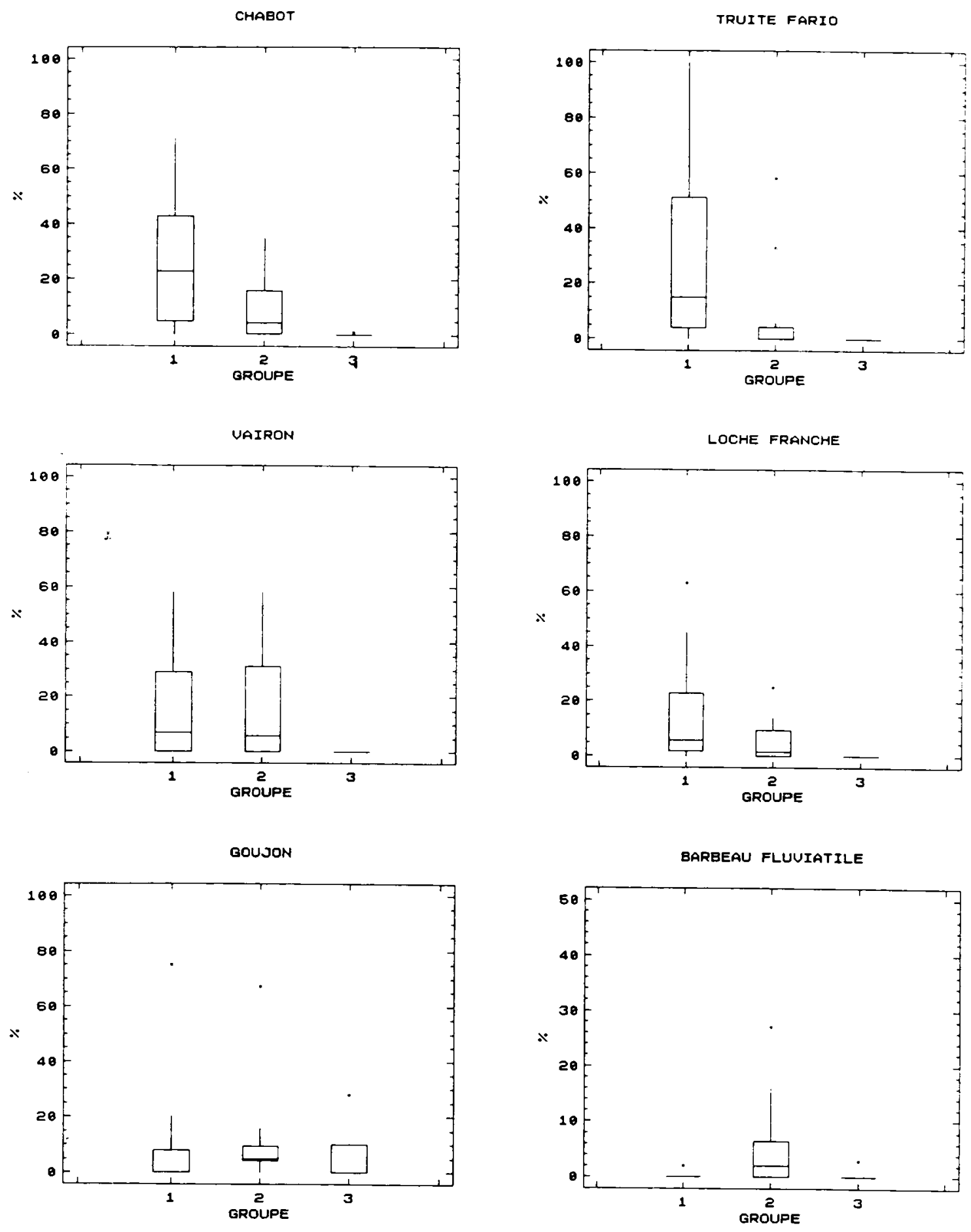
Bull. Fr. Pêche Piscic. (1991) $320: 7-28 \quad-23-$
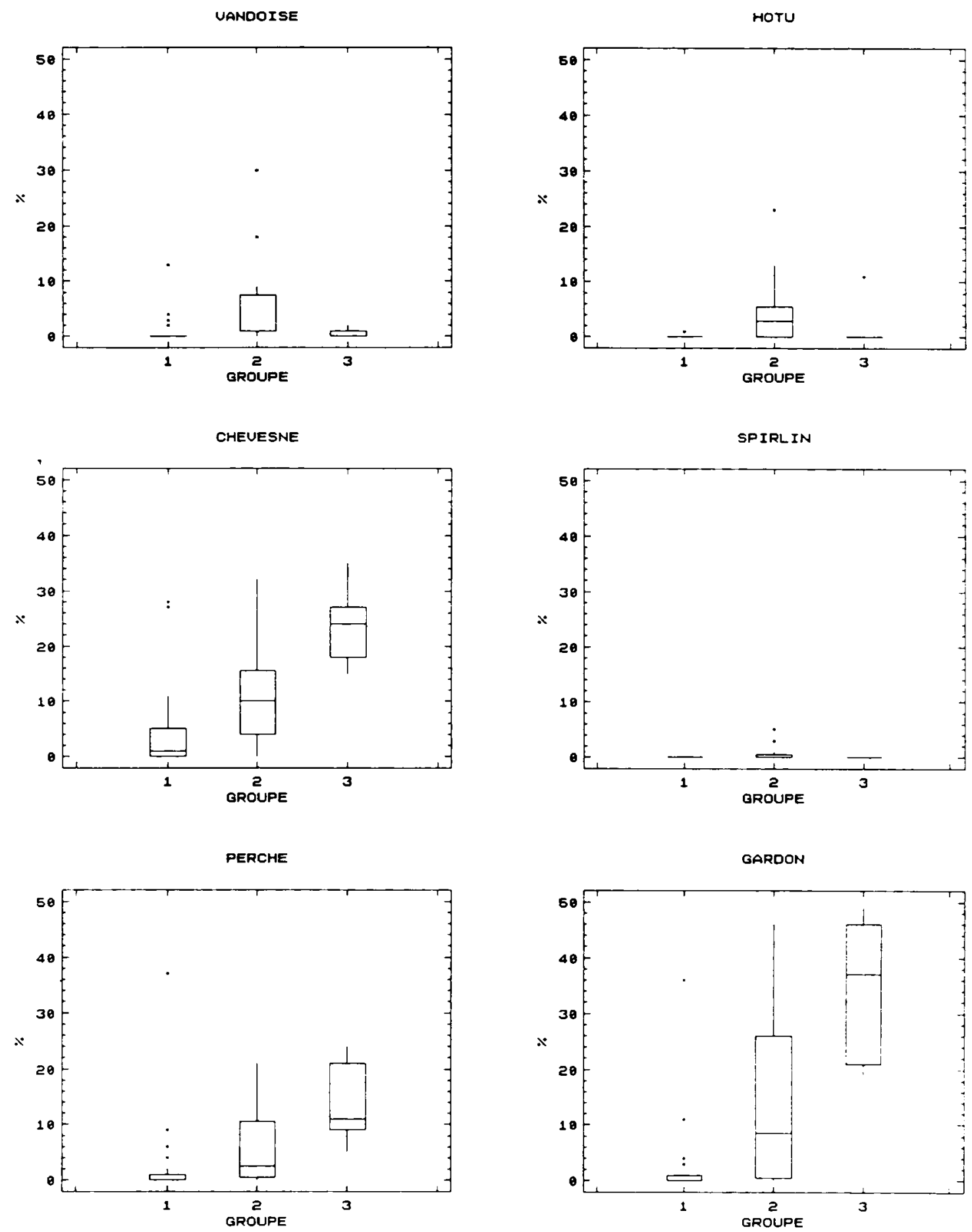

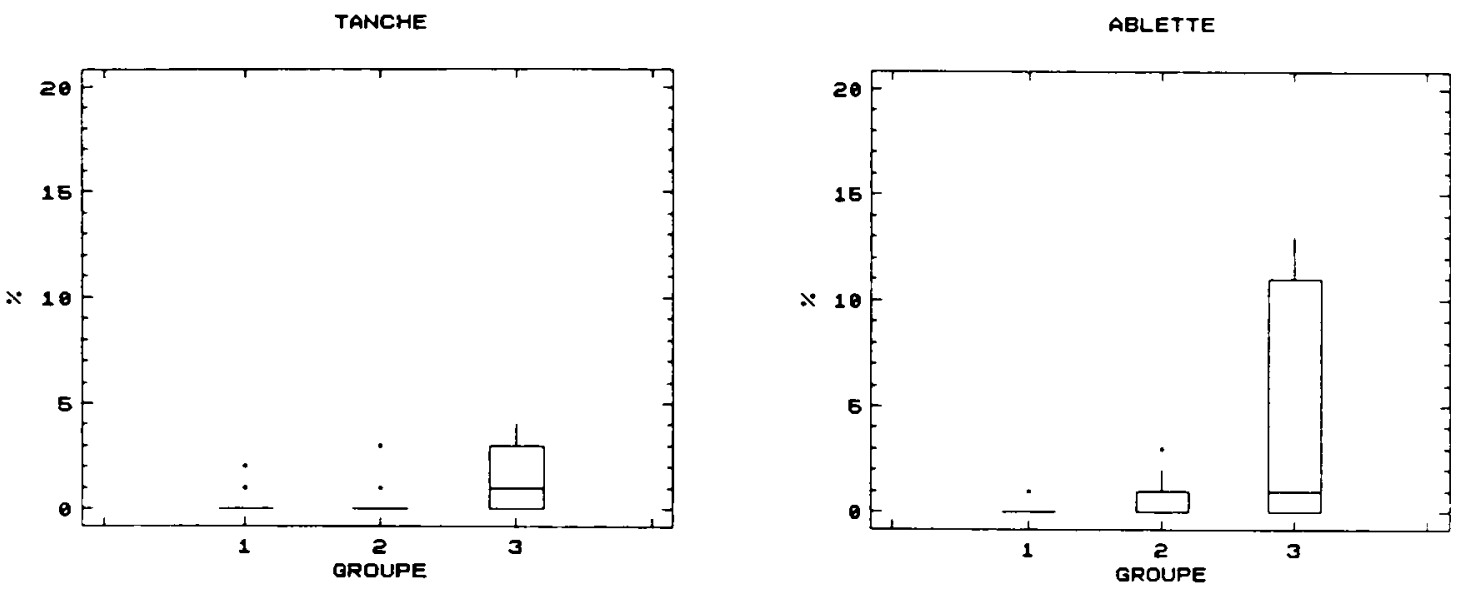

BROCHET

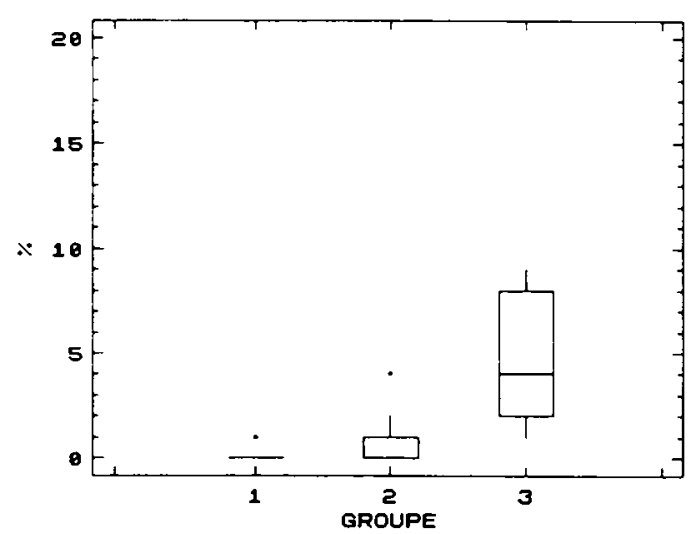

BREME BORDELIERE

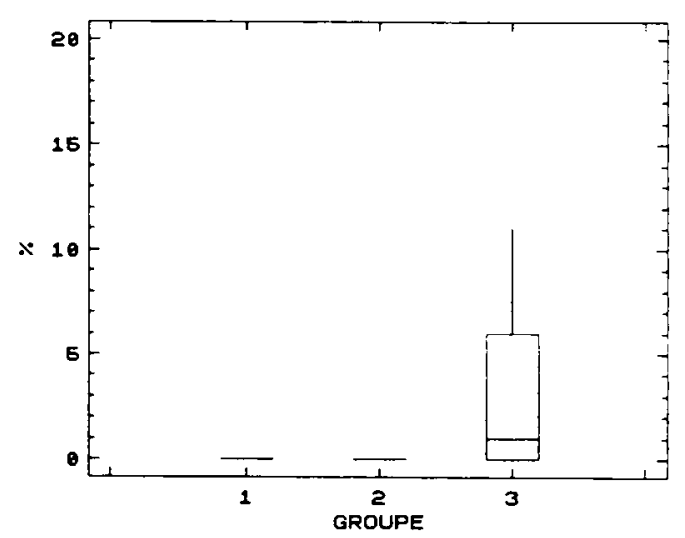

ROTANGLE
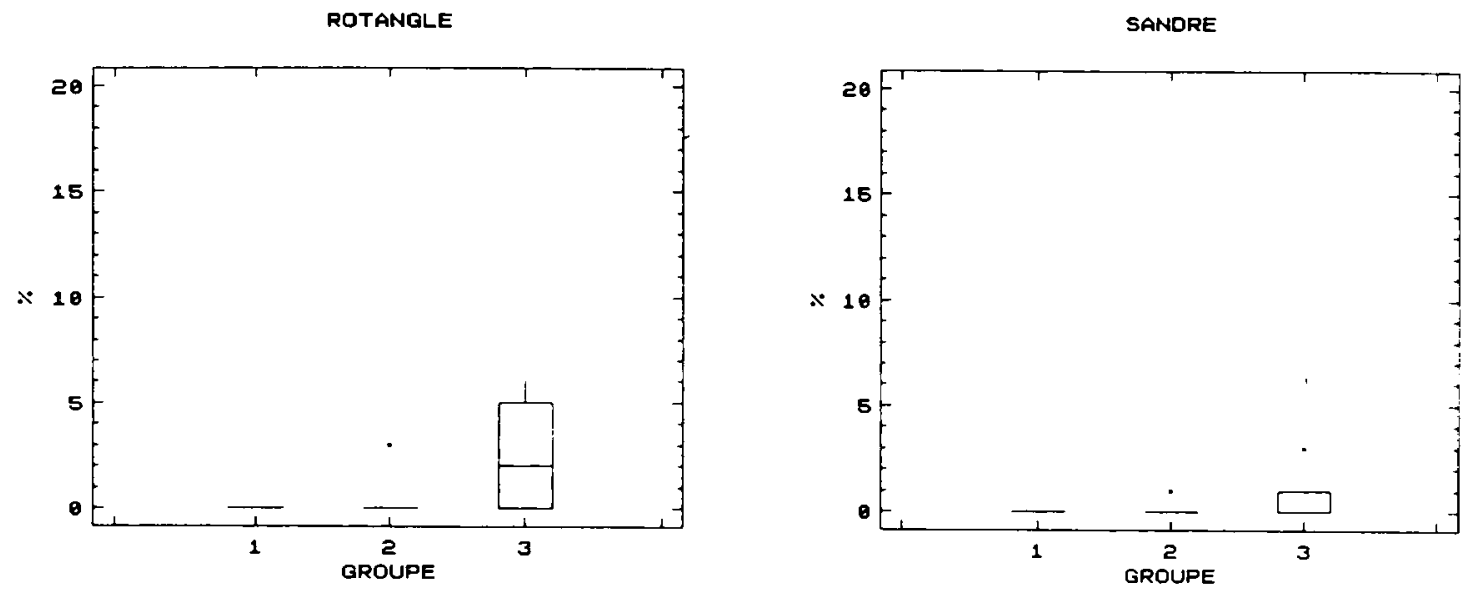


\section{DISCUSSION ET CONCLUSIONS}

Sur le cours principal de l'Yonne, l'évolution de la composition spécifique du peuplement est conforme aux modèles théoriques, depuis la source jusqu'au niveau de Surgy et, à un degré moindre, Appoigny. Celle-ci consiste en une augmentation continue de la richesse spécifique. Cette augmentation s'effectue par le remplacement progressif de la Truite et de ses espèces d'accompagnement (Chabot, Vairon, Loche franche) par des cyprinidés rhéophiles (Barbeau, Vandoise, Hotu assez rare ici) et l'apparition d'espèces lénitophiles et phytotoques (Carpe, Tanche, Brochet, Rotengle...).

Mais, tandis que la qualité de l'eau devrait normalement permettre à toutes ces espèces d'accomplir leur cycle biologique sur l'ensemble du cours d'eau puis, à l'aval de Montereau, dans la Seine, force est de constater la nette réduction de la diversité spécifique dès que sont atteints les secteurs navigués et chenalisés.

Les différents aménagements du cours d'eau, nécessaires à la navigation, entraînent en effet une forte régression des faciès lotiques, voire leur disparition complète, sauf à l'aval immédiat des barrages. De cette dégradation des habitats, il résulte une diminution sensible des cyprinidés rhéophiles, illustrée par leurs faibles fréquences de captures.

Ces observations sont confirmées par la prise en compte des résultats de pêches effectuées sur un grand nombre d'affluents (20 au total). Leur analyse confrontant la composition du peuplement échantillonné avec les caractéristiques des stations permet en effet de dégager nettement, à l'échelle du bassin, un schéma analogue d'organisation et d'évolution des peuplements.

En une station donnée, la composition du peuplement dépend de paramètres topographiques et morphodynamiques à évolution continue ; la chenalisation est un facteur d'uniformisation. La répartition des espèces s'effectue suivant un gradient dont la distance à la source, l'altitude et l'ordre du cours d'eau au niveau de la station sont en moyenne représentatifs.

Cette organisation se trouve perturbée, d'une part, par la présence sur les stations amont d'espèces "parasites" (Gardon, Perche, Brochet) provenant des multiples étangs du Morvan et, d'autre part, par la simplification des habitats et des biocénoses dans les secteurs navigués.

A ces modifications physiques néfastes des habitats s'ajoutent probablement les conséquences de l'épizootie de Bucéphalose larvaire, due au ver Trématode parasite (Bucephalus polymorphus). Mise en évidence sur le bassin de la Seine (DE KINKELIN et al., 1968), elle sévit depuis plus de vingt ans et provoque des mortalités importantes qui peuvent passer inaperçues lorsqu'elles ne touchent que les alevins.

Les faibles densités de poissons capturés au cours des pêches de juin 1988 , particulièrement sur les stations aval, peuvent être la conséquence de cette maladie ; celle-ci est accentuée par l'aménagement de la rivière (chenalisation), favorable aux développements de la moule d'eau douce (Dreissena polymorpha) et du Sandre, les deux hôtes indispensables à la réalisation du cycle parasitaire.

La comparaison de ces résultats avec les données anciennes de MOREAU (1987, 1988) ou de POPELIN (1952) permet d'apprécier l'évolution récente de l'Yonne au plan piscicole et de mesurer l'impact des perturbations d'origine anthropique sur le milieu.

Les nombreux aménagements anciens destinés à la navigation, qui constituent des obstacles infranchissables pour les poissons, ont provoqué la disparition de la plupart des espèces migratrices.

Au siècle dernier, l'Esturgeon n'était que rarement observé jusqu'à Auxerre mais il y était pêché quelquefois. L'Alose, bien que paraissant déjà rare en 1898, remontait autrefois l'Yonne jusqu'à Auxerre.

Le Saumon, plus commun, empruntait généralement la Cure et parvenait jusqu'aux étangs des Settons (Nièvre). Quelques individus remontaient l'Armançon, d'autres, qui ne quittaient pas l'Yonne, avaient été capturés aux environs de Corbigny. Dans le cas particulier de l'Yonne, l'extinction du Saumon est ancienne, puisqu'elle remonte 
probablement aux premiers aménagements réalisés pour les besoins du flottage du bois (barrage des Settons, 1858), qui ont précédé ceux nécessités pour la navigation (LAVOLLÉE, 1902).

Enfin, bien qu'observée deux fois au cours des inventaires réalisés en juin 1988, il semble que l'anguille ait été plus fréquente jadis (MOREAU, 1898).

La chenalisation entraînant la disparition des radiers indispensables au dépôt des œufs chez les espèces rhéophiles, explique la diminution de certains Cyprinidés comme le Spirlin ou la Vandoise, naguère bien représentés.

La régression de la Bouvière (ARCHAMBAULT, 1986) est sans doute également due à son mode de reproduction très particulier (ponte des œufs à l'intérieur de moules de la famille des Unionidés).

Au contraire, cinq espèces nouvelles s'ajoutent au peuplement de l'Yonne, la Truite Arc-en-Ciel, l'Ombre commun, le Poisson-Chat, la Perche Soleil et le Sandre. La Truite Arcen-Ciel et l'Ombre commun ne sont représentés que par un très faible nombre d'individus. Introduit en France vers 1870, le Poisson-Chat, mentionné dans les inventaires du C.S.P. en zones amont, à Montreuillon et à Corbigny, ne paraît pas s'être développé depuis. En revanche, la Perche Soleil est signalée dans plusieurs stations et le Sandre, dont l'introduction en France remonte au début du siècle (1925), semble se maintenir dans les secteurs calmes de la partie aval du cours d'eau, où il a été introduit au début des années 1960 (GOUBIER, 1975).

31 espèces sont maintenant recensées sur l'ensemble du bassin, chiffre élevé et proche d'une richesse spécifique globale maximum pour cette région biogéographique.

Mais cependant, cette étude du peuplement piscicole du bassin de I'Yonne conduit à mettre en évidence une nette discordance entre la qualité physico-chimique relativement satisfaisante du milieu aquatique et l'importante composante biologique constituée par le peuplement des poissons.

Une situation similaire semble se retrouver sur l'ensemble du bassin de la Seine, dans les secteurs navigués. Dans ce type de contexte, les actions de réhabilitation ne doivent donc pas porter uniquement sur l'amélioration de la qualité physico-chimique des eaux ; elles doivent également viser à préserver ou restaurer l'habitat de la faune piscicole.

\section{REMERCIEMENTS}

Les auteurs remercient Evelyne TALES, B. DUVOUX et A. RUMEAU pour leur collaboration dans la réalisation de cette étude qui a fait l'objet d'une convention entre l'Agence Financière de Bassin Seine-Normandie et le CEMAGREF.

\section{BIBLIOGRAPHIE}

ALATALO R.V., 1981. Problems in the measurement of evenness in ecology. Oikos, 37, 199-204.

ALATALO R.V. et ALATALO R., 1977. Components of diversity : multivariate analysis with interaction. Ecology, 58, 900-906.

ARCHAMBAULT Ph., 1986. Évolution des principales espèces de poissons dans I'Yonne de Paul Bert à nos jours. in C.R.D.P., Ed. Le catalogue des Vertébrés de l'Yonne de Paul Bert. Réédition augmentée, Dijon, $150 \mathrm{p}$.

ARRIGNON J., 1988. The Loire. Sciences de l'eau, 7 (1), 21-34.

BENZECRI J.-P. et Collaborateurs, 1984. L'analyse des données I : taxinomie, /I : L'analyse des correspondances. $4^{e}$ éd. Dunod Paris, 635 et $632 \mathrm{p}$.

CEMAGREF, 1989. Étude Hydrobiologique et piscicole de l'Yonne. Tome I : Rapport de synthèse, Tome II : Annexes cartographiques. Rapp. D.Q.E.P.P. Antony - A.F.B. Seine-Normandie. Novembre 1989, 83 p. + annexes. 
DAGET J., 1980. Le nombre de diversité de HILL, un concept unificateur dans la théorie de la diversité écologique. Acta Oecologica/Oecol. Gener., 1, 51-70.

DE KINKELIN P., TUFFERY G., LEYNAUD G. et ARRIGNON J., 1968. Étude épizootiologique de la bucéphalose larvaire à Bucephalus polymorphus Baer (1827) dans le peuplement piscicole du bassin de la Seine. Rech. vétér., 1, 77-98.

GOODNIGHT C.H., 1973. The use of aquatic macroinvertebrates as indicators of stream pollution. Trans Amer. Micros. Soc., 92 (1), 1-13.

GOUBIER J., 1975. Biogéographie, biométrie et biologie du Sandre, Lucioperca lucioperca (L.), Osteichthyen, Percidé. Thèse Doc. État ès-Sciences Univ. Claude Bernard Lyon I, $259 \mathrm{p}$.

HILL M.O., 1973. Diversity and evenness : a unifying notation and its consequences. Ecology, 54, 427-432.

HORTON R.E., 1945. Erosional development of streams and their drainage basins hydrophysical approach to quantitative morphology. Bull. Geol. Soc. Am., 56, 275-370.

HUET M., 1949. Aperçu des relations entre la pente et les populations piscicoles des eaux courantes. Scheiw. Z. Hydrol., 11 (3-4), 332-351.

ILLIES J., et BOTOSANEANU L., 1963. Problèmes et méthodes de la classification et de la zonation écologique des eaux courantes, considérées surtout du point de vue faunistique. Mitt. Intern. Ver. Limnol., 12, 1-57.

JUAN J., 1984. Programme de classification ascendante hiérarchique selon les voisins réciproques et le critère de variance. in BENZECRI J.-P. et Collaborateurs, 1984. L'analyse des données I : taxinomie, 4e éd. Dunod Paris, 569-580.

KUEHNE R.A., 1962. A classification of streams, illustrated by fish distribution in an eastern Kentucky Creek. Ecology, 43 (4), 608-614.

LAVOLlEE M.G., 1902. Le Saumon en Seine. Bull. Soc. Centrale Aquicult. et Pêche, 14, 221-234.

LEGENDRE L. et LEGENDRE P., 1984. Écologie numérique. Tome 1 : Le traitement multiple des données écologiques. (2e éd.), Coll. Écol. 12, Masson Paris, 260 p.

LUDWIG J.A. et REYNOLDS J.F., 1988. Statistical Ecology. A primer on methods and computing. John Willey and Sons, New York, $337 \mathrm{p}$.

MOLINARI J., 1989. A calibrated index for the measurement of evenness. Oikos, 56, 319326.

MOREAU E., 1897. Les poissons du département de I'Yonne. Bull Soc. Sci. Hist. Nat. Yonne, vol. 51 part 2, 143-227.

MOREAU E., 1898. Les poissons du département de I'Yonne. Bull Soc. Sci. Hist. Nat. Yonne, vol. 52 part 2, 3-82.

PATTEE E., 1988. The Rhône. Sciences de l'Eau, 7 (1), 35-74.

PEET R.K., 1974. The measurement of species diversity. Annual Rev. Acol. Syst., 5, 285307.

POPELIN R., 1952. Le peuplement des eaux de I'Yonne moyenne. Bull. Fr. Piscicult., 164, 109-114.

RENYI A., 1961. On measures of entropy and information. in Neyman J., Éd. Proc. 4th. Berkeley Symp. on mathematical statistics and probability. Berkeley, 547-561.

SHANNON C.E., 1948. A mathematical theory of communications. Bell System Technical Jour., 27, 379-423, 623-656.

SIMPSON E.H., 1949. Measurement of diversity. Nature, 163, 688.

STRAHLER A.N., 1957. Quantitative analysis of watershed geomorphology. Trans. Amer. Geophys. Union, 38, 913-920. 
VERNEAUX J., 1973. Cours d'eau de Franche-Comté (massif du Jura). Recherches écologiques sur le réseau hydrographique du Doubs. Essai de biotypologie. Thèse État Doc. ès-Sciences Univ. Besançon. Ann. Fac. Sci. Univ. Besançon n 84, 257 p.

VERNEAUX J., 1977. Biotypologie de l'écosystème "eau courante". Déterminisme approché de la structure biotypologique. C.R. Acad. Sc. Paris, t. 284 sér. D, 77-79.

VERNEAUX J., 1981. Les poissons et la qualité des cours d'eau. Ann. Sci. Univ. FrancheComté Besançon, Biol. Anim., $4^{e}$ sér. fasc. 2, 33-41. 